\title{
Multigrid Optimization for Space-Time Discontinuous Galerkin Discretizations of Advection Dominated Flows
}

S. Rhebergen, J.J.W. van der Vegt and H. van der Ven

\begin{abstract}
The goal of this research is to optimize multigrid methods for higher order accurate space-time discontinuous Galerkin discretizations. The main analysis tool is discrete Fourier analysis of two- and three-level multigrid algorithms. This gives the spectral radius of the error transformation operator which predicts the asymptotic rate of convergence of the multigrid algorithm. In the optimization process we therefore choose to minimize the spectral radius of the error transformation operator. We specifically consider optimizing $h$-multigrid methods with explicit RungeKutta type smoothers for second and third order accurate space-time discontinuous Galerkin finite element discretizations of the 2D advection-diffusion equation. The optimized schemes are compared with current $h$-multigrid techniques employing Runge-Kutta type smoothers. Also, the efficiency of $h$-, $p$ - and $h p$-multigrid methods for solving the Euler equations of gas dynamics with a higher order accurate space-time DG method is investigated.
\end{abstract}

\section{Introduction}

Space-time discontinuous Galerkin (DG) discretizations of time-dependent partial differential equations result in a system of (non)-linear algebraic equations which can be solved efficiently with multigrid methods. In this paper we will discuss the optimization of multigrid techniques for higher order accurate space-time DG discretizations describing advection dominated flows. This research is a continuation of $[3,7]$ where we presented a multigrid algorithm in combination with a pseudo-time

S. Rhebergen, J.J.W. van der Vegt

University of Twente, Department of Applied Mathematics, P.O. Box 217, 7500 AE, Enschede, The Netherlands, e-mail: $\{$ s.rhebergen, j.j.w.vandervegt $\}$ math.utwente.nl

H. van der Ven

National Aerospace Laboratory NLR, P.O. Box 90502, 1006 BM, Amsterdam, The Netherlands, e-mail: venvd@nlr.nl 
integration method for second order accurate space-time DG discretizations of the compressible Euler and Navier-Stokes equations. The main benefits of this multigrid algorithm are that no large global linear system needs to be solved and, through the use of Runge-Kutta type smoothers, the locality of the DG discretization is preserved. The algorithm is easy to implement and parallelize, even on locally refined meshes, and insensitive to initial conditions. For higher order accurate space-time DG discretizations the multigrid performance was, however, not satisfactory. The objective of this paper is to discuss improvements in the computational performance of space-time DG discretizations when higher order polynomial basis functions are used. The main tool to analyze the multigrid performance is three-level discrete Fourier analysis. This analysis tool is used to optimize the multigrid performance by minimizing the spectral radius of the multigrid error transformation operator. In particular, the focus will be on searching for better coefficients in the multigrid smoothing operator. More detailed information on the multigrid algorithms and the analysis techniques used in this paper can be found in e.g. [1, 6, 10,11].

The outline of this paper is as follows. After a brief introduction in Section 2 on the multigrid error transformation operator, a summary of the discrete Fourier analysis of the multigrid algorithm will be given in Section 3. Next, we discuss the optimization of the multigrid algorithm in Section 4. Results of the optimization process will be given in Section 5 as well as a comparison in efficiency between $h$-, $p$ - and $h p$-multigrid methods. Finally, conclusions are drawn in Section 6.

\section{Multigrid error transformation operator}

The main goal of the multigrid algorithm is to iteratively solve in an efficient way a system of (non)-linear algebraic equations $L_{h} v_{h}=f_{h}$ on a mesh $\mathscr{M}_{h}$, with $L_{h}$ a linear or non-linear discretization operator and $f_{h}$ a given righthand side. In the $h$ multigrid method we use a finite sequence $N_{c}$ of increasingly coarser meshes $\mathscr{M}_{n h}$, $n \in\left\{1, \cdots, N_{c}\right\}$ to generate approximations to the original problem. In addition, the data on the different meshes are connected with restriction operators $R_{n h}^{m h}: \mathscr{M}_{n h} \rightarrow$ $\mathscr{M}_{m h}$ and prolongation operators $P_{m h}^{n h}: \mathscr{M}_{m h} \rightarrow \mathscr{M}_{n h}$, with $1 \leq n<m \leq N_{c}$. On these meshes a set of auxiliary problems is solved $\mathscr{M}_{n h}, 1<n \leq N_{c}$, namely $L_{n h} v_{n h}=f_{n h}$, in order to accelerate convergence. For non-linear problems we use the Full Approximation Scheme (FAS), see e.g. [6], but in the analysis of the multigrid performance we only consider linear problems.

In order to understand the performance of the multigrid algorithm we need to consider the multigrid error transformation operator. Given an initial error $e_{h}^{A}$, the error $e_{h}^{D}$ after one full multigrid cycle with three grid levels is given by the relation

$$
e_{h}^{D}=M_{h}^{3 g} e_{h}^{A}
$$

with

$$
M_{h}^{3 g}=S_{h}^{V_{2}}\left(I_{h}-P_{2 h}^{h}\left(I_{2 h}-M_{2 h}^{\gamma_{c}}\right) L_{2 h}^{-1} R_{h}^{2 h} L_{h}\right) S_{h}^{\nu_{1}}
$$


and

$$
M_{2 h}=S_{2 h}^{V_{4}}\left(I_{2 h}-P_{4 h}^{2 h} L_{4 h}^{-1} R_{2 h}^{4 h} L_{2 h}\right) S_{2 h}^{\nu_{3}}
$$

Here, $S_{n h}$ and $I_{n h}$ are, respectively, the smoothing and identity operator on the mesh $\mathscr{M}_{n h}, \quad v_{i}, i=1, \cdots, 4$, the number of pre- and post-smoothing iterations and $\gamma_{c}$ the cycle index. In the multigrid analysis and computations we will also consider the effect of solving the algebraic system on the coarsest mesh approximately using $v_{c}$ smoother iterations instead of using an exact inverse. Next to $h$-multigrid also $p$ multigrid methods are possible in which on a single mesh coarser approximations are obtained by using lower order discretizations. Of course, combinations of both techniques are possible resulting in $h p$-multigrid methods.

\section{Three-level multigrid analysis}

\subsection{Discrete Fourier analysis}

Consider the infinite mesh $G_{h}$, which is defined as

$$
G_{h}:=\left\{x=\left(x_{1}, x_{2}\right)=\left(k_{1} h_{1}, k_{2} h_{2}\right) \mid k \in \mathbb{Z}^{2}, h \in\left(\mathbb{R}^{+}\right)^{2}\right\} .
$$

On $G_{h}$ we define for $v_{h}: G_{h} \rightarrow \mathbb{C}$ the norm

$$
\left\|v_{h}\right\|_{G_{h}}^{2}:=\lim _{N \rightarrow \infty} \frac{1}{4 N^{2}} \sum_{|k| \leq m}\left|v_{h}(k h)\right|^{2}
$$

where $|k|=\max \left\{\left|k_{1}\right|,\left|k_{2}\right|\right\}$. In the theoretical analysis we only consider linear problems, where the linear systems on the various meshes are described using stencil notation

$$
L_{n h} v_{n h}(x)=\sum_{k \in J_{n}} l_{n, k} v_{n h}(x+k h), \quad x \in G_{n h},
$$

with stencil coefficients $l_{n, k} \in \mathbb{R}^{m_{k} \times m_{k}}$ and finite index sets $J_{n} \subset \mathbb{Z}^{2}$ describing the stencil. The restriction operators $R_{n h}^{m h}$, prolongation operators $P_{m h}^{n h}$ and smoothing operators $S_{n h}$ with $1 \leq n<m \leq N_{c}$ are also expressed using stencil notation, see e.g. $[6,10,11]$.

On the infinite mesh $G_{h}$, we define for $x \in G_{h}$ the continuous Fourier modes with frequency $\theta=\left(\theta_{1}, \theta_{2}\right) \in \mathbb{R}^{2}$ as $\phi_{h}(\theta, x):=e^{i \theta \cdot x / h}$ with $\theta \cdot x / h:=\theta_{1} x_{1} / h_{1}+\theta_{2} x_{2} / h_{2}$, $h \in\left(\mathbb{R}^{+}\right)^{2}$ and $i=\sqrt{-1}$. We also define the space of bounded infinite grid functions by $\mathscr{F}\left(G_{h}\right):=\left\{v_{h} \mid v_{h}: G_{h} \rightarrow \mathbb{C}\right.$ with $\left.\left\|v_{h}\right\|_{G_{h}}<\infty\right\}$. For each $v_{h} \in \mathscr{F}\left(G_{h}\right)$ there exists a Fourier transformation, hence $v_{h}(x)$ can be written as a linear combination of Fourier components

$$
v_{h}(x)=\int_{|\theta| \leq \pi} \widehat{v_{h}}(\theta) e^{i \theta \cdot x / h} d \theta, \quad x \in G_{h},
$$


with $x / h:=\left(x_{1} / h_{1}, x_{2} / h_{2}\right)=j \in \mathbb{Z}^{2}$, and inverse transformation

$$
\widehat{v_{h}}(\theta)=\frac{1}{4 \pi^{2}} \sum_{x \in G_{h}} v_{h}(x) e^{-i \theta \cdot x / h}, \quad-\pi \leq \theta_{j}<\pi,
$$

see e.g. [1]. Due to aliasing, Fourier components with $|\hat{\theta}|:=\max \left\{\left|\theta_{1}\right|,\left|\theta_{2}\right|\right\} \geq \pi$ are not visible on $G_{h}$. These modes coincide with $e^{i \theta \cdot x / h}$, where $\theta=\hat{\theta}(\bmod 2 \pi)$. Hence, the Fourier space $\mathscr{F}:=\operatorname{span}\left\{e^{i \theta \cdot x / h} \mid \theta \in \Theta=[-\pi, \pi)^{2}, x \in G_{h}\right\}$ contains any bounded infinite grid function.

\subsection{Three-grid Fourier analysis}

For the three-grid Fourier analysis we define the Fourier harmonics $\mathscr{F}_{4 h}(\theta)$ as

$$
\begin{aligned}
& \mathscr{F}_{4 h}(\theta):=\operatorname{span}\left\{\phi_{h}\left(\theta_{\beta}^{\alpha}, x\right) \mid \alpha \in \alpha_{2}, \beta \in \beta_{2}\right\}, \quad \text { where } \\
& \theta=\theta_{0}^{0} \in \Theta_{4 h}:=[-\pi / 4, \pi / 4)^{2}, \\
& \theta_{\beta}=\theta_{0}^{0}-\left(\bar{\beta}_{1} \operatorname{sign}\left(\theta_{1}\right), \bar{\beta}_{2} \operatorname{sign}\left(\theta_{2}\right)\right) \pi, \\
& \theta_{\beta}^{\alpha}:=\theta_{\beta}-\left(\bar{\alpha}_{1} \operatorname{sign}\left(\left(\theta_{1}\right)_{\beta}\right), \bar{\alpha}_{2} \operatorname{sign}\left(\left(\theta_{2}\right)_{\beta}\right)\right) \pi, \\
& \alpha_{2}:=\left\{\alpha=\left(\bar{\alpha}_{1}, \bar{\alpha}_{2}\right) \mid \bar{\alpha}_{i} \in\{0,1\}, i=1,2\right\} \\
& \beta_{2}:=\left\{\beta=\left(\bar{\beta}_{1}, \bar{\beta}_{2}\right) \mid \bar{\beta}_{i} \in\left\{0, \frac{1}{2}\right\}, i=1,2\right\} .
\end{aligned}
$$

Note that we have 16 coupled Fourier harmonics, all related to $\theta_{00}^{00}$. In the transition from $G_{2 h}$ to $G_{4 h}$ the modes $\theta_{\beta}=\theta_{\beta}^{0}$ are not visible due to aliasing.

The error $e_{h}^{D}$ after one iteration of a three-grid multigrid cycle is determined by $e_{h}^{D}=M_{h}^{3 g} e_{h}^{A}$, with $e_{h}^{A}$ the initial error and $M_{h}^{3 g}$ the three-level multigrid error transformation operator defined by (1).

The properties of the error transformation operator can be investigated using discrete Fourier analysis. For this purpose we introduce the following matrices

$$
\begin{aligned}
& \widehat{L}_{h}^{2 g}\left(\theta_{\beta}\right)=\operatorname{diag}\left(\widehat{L_{h}}\left(\theta_{\beta}^{00}\right), \widehat{L_{h}}\left(\theta_{\beta}^{11}\right), \widehat{L_{h}}\left(\theta_{\beta}^{10}\right), \widehat{L_{h}}\left(\theta_{\beta}^{01}\right)\right) \in \mathbb{C}^{4 m \times 4 m} \\
& \widehat{S}_{h}^{2 g}\left(\theta_{\beta}\right)=\operatorname{diag}\left(\widehat{S_{h}}\left(\theta_{\beta}^{00}\right), \widehat{S_{h}}\left(\theta_{\beta}^{11}\right), \widehat{S_{h}}\left(\theta_{\beta}^{10}\right), \widehat{S_{h}}\left(\theta_{\beta}^{01}\right)\right) \in \mathbb{C}^{4 m \times 4 m} \\
& \widehat{R}_{h}^{2 g}\left(\theta_{\beta}\right)=\left(\widehat{R_{h}^{2 h}}\left(\theta_{\beta}^{00}\right), \widehat{R_{h}^{2 h}}\left(\theta_{\beta}^{11}\right), \widehat{R_{h}^{2 h}}\left(\theta_{\beta}^{10}\right), \widehat{R_{h}^{2 h}}\left(\theta_{\beta}^{01}\right)\right) \in \mathbb{C}^{m \times 4 m} \\
& \widehat{P}_{h}^{2 g}\left(\theta_{\beta}\right)=\left(\widehat{P_{2 h}^{h}}\left(\theta_{\beta}^{00}\right), \widehat{P_{2 h}^{h}}\left(\theta_{\beta}^{11}\right), \widehat{P_{2 h}^{h}}\left(\theta_{\beta}^{10}\right), \widehat{P_{2 h}^{h}}\left(\theta_{\beta}^{01}\right)\right)^{T} \in \mathbb{C}^{4 m \times m}
\end{aligned}
$$

where diag refers to a diagonal matrix consisting of $m \times m$ blocks with $m \in \mathbb{N}$. The Fourier symbol of the linear operator $L_{n h}$ is equal to $\widehat{L_{n h}}(\theta)=\sum_{k \in J_{n}} l_{n, k} e^{i \theta \cdot k}$. Similar expressions can be derived for the Fourier symbols of the restriction operator $\widehat{R_{n h}^{m h}}(\theta)$, the prolongation operator $\widehat{P_{m h}^{n h}}(\theta)$ and the smoothing operator $\widehat{S_{n h}}(\theta)$ on 
the various mesh levels. For more details, see e.g. $[1,6,11]$. We also introduce the matrices

$$
\begin{aligned}
& \widehat{L}_{h}^{3 g}(\theta)=\operatorname{bdiag}\left(\widehat{L}_{h}^{2 g}\left(\theta_{00}\right), \widehat{L}_{h}^{2 g}\left(\theta_{\frac{1}{2} \frac{1}{2}}\right), \widehat{L}_{h}^{2 g}\left(\theta_{\frac{1}{2} 0}\right), \widehat{L}_{h}^{2 g}\left(\theta_{0 \frac{1}{2}}\right)\right) \in \mathbb{C}^{16 m \times 16 m} \\
& \widehat{S}_{h}^{3 g}(\theta)=\operatorname{bdiag}\left(\widehat{S}_{h}^{2 g}\left(\theta_{00}\right), \widehat{S}_{h}^{2 g}\left(\theta_{\frac{1}{2} \frac{1}{2}}\right), \widehat{S}_{h}^{2 g}\left(\theta_{\frac{1}{2}}\right), \widehat{S}_{h}^{2 g}\left(\theta_{0 \frac{1}{2}}\right)\right) \in \mathbb{C}^{16 m \times 16 m} \\
& \widehat{R}_{h}^{3 g}(\theta)=\operatorname{bdiag}\left(\widehat{R}_{h}^{2 g}\left(\theta_{00}\right), \widehat{R}_{h}^{2 g}\left(\theta_{\frac{1}{2} \frac{1}{2}}\right), \widehat{R}_{h}^{2 g}\left(\theta_{\frac{1}{2} 0}\right), \widehat{R}_{h}^{2 g}\left(\theta_{0 \frac{1}{2}}\right)\right) \in \mathbb{C}^{4 m \times 16 m} \\
& \widehat{P}_{h}^{3 g}(\theta)=\operatorname{bdiag}\left(\widehat{P}_{h}^{2 g}\left(\theta_{00}\right), \widehat{P}_{h}^{2 g}\left(\theta_{\frac{1}{2} \frac{1}{2}}\right), \widehat{P}_{h}^{2 g}\left(\theta_{\frac{1}{2} 0}\right), \widehat{P}_{h}^{2 g}\left(\theta_{0 \frac{1}{2}}\right)\right) \in \mathbb{C}^{16 m \times 4 m} \\
& \widehat{Q}_{h}^{3 g}(\theta)=\operatorname{bdiag}\left(\widehat{L_{2 h}^{-1}}\left(2 \theta_{00}\right), \widehat{L_{2 h}^{-1}}\left(2 \theta_{\frac{1}{2} \frac{1}{2}}\right) \widehat{L_{2 h}^{-1}}\left(2 \theta_{\frac{1}{2} 0}\right), \widehat{L_{2 h}^{-1}}\left(2 \theta_{0 \frac{1}{2}}\right)\right) \in \mathbb{C}^{4 m \times 4 m} .
\end{aligned}
$$

The discrete Fourier transform of the error transformation operator for a three-level multigrid cycle $\widehat{M}_{h}^{3 g}(\theta) \in \mathbb{C}^{16 m \times 16 m}$ then is equal to [11]

$$
\widehat{M}_{h}^{3 g}(\theta)=\left(\widehat{S}_{h}^{3 g}(\theta)\right)^{v_{2}}\left(I^{3 g}-\widehat{P}_{h}^{3 g}(\theta) \widehat{U}^{3 g}\left(\theta ; \gamma_{c}\right) \widehat{Q}_{h}^{3 g}(\theta) \widehat{R}_{h}^{3 g}(\theta) \widehat{L}_{h}^{3 g}(\theta)\right)\left(\widehat{S}_{h}^{3 g}(\theta)\right)^{v_{1}}
$$

with $I^{3 g}$ the $16 m \times 16 m$ identity matrix and $\theta \in \Theta_{4 h} \backslash \Psi_{3 g}$, where $\Psi_{3 g}$ is defined as $\Psi_{3 g}:=\left\{\theta \in \Theta_{4 h} \mid \widehat{L_{4 h}}\left(4 \theta_{0}^{0}\right)=0\right.$ or $\widehat{L_{2 h}}\left(2 \theta_{\beta}^{0}\right)=0$ or $\left.\widehat{L_{h}}\left(\theta_{\beta}^{\alpha}\right)=0\right\}$. We still need to obtain an explicit expression for $\widehat{U}^{3 g}\left(\theta ; \gamma_{c}\right) \in \mathbb{C}^{4 m \times 4 m}$. On the mesh $G_{2 h}$ the modes $\theta_{\beta}^{\alpha}$ reduce after the restriction operator to modes $2 \theta_{\beta}^{0}$, hence using the result of a two-level analysis the coarse grid error transformation operator is equal to

$\widehat{M}_{2 h}^{2 g}\left(2 \theta_{\beta}\right)=\left(\widehat{S}_{2 h}^{2 g}\left(2 \theta_{\beta}\right)\right)^{v_{4}}\left(I^{2 g}-\widehat{P}_{2 h}^{2 g}\left(2 \theta_{\beta}\right) \widehat{L}_{4 h}^{-1}\left(4 \theta_{0}^{0}\right) \widehat{R}_{2 h}^{2 g}\left(2 \theta_{\beta}\right) \widehat{L}_{2 h}^{2 g}\left(2 \theta_{\beta}\right)\right)\left(\widehat{S}_{2 h}^{2 g}\left(2 \theta_{\beta}\right)\right)^{v_{3}}$,

with $I^{2 g}$ the $4 m \times 4 m$ identity matrix and $\theta_{\beta} \in \Theta_{2 h}:=[-\pi / 4, \pi / 4)^{2} \backslash \Psi_{2 g}$, where $\Psi_{2 g}$ is defined as $\Psi_{2 g}:=\left\{\theta \in[-\pi / 4, \pi / 4)^{2} \mid \widehat{L_{4 h}}\left(4 \theta_{0}^{0}\right)=0\right.$ or $\left.\widehat{L_{2 h}}\left(2 \theta_{\beta}^{0}\right)=0\right\}$. The matrices $\widehat{L}_{2 h}^{2 g}, \widehat{S}_{2 h}^{2 g}, \widehat{R}_{2 h}^{2 g}$ and $\widehat{P}_{2 h}^{2 g}$ are given by (5)-(8), respectively, with $h$ replaced by $2 h$. The matrix $\widehat{U}^{3 g}\left(\theta ; \gamma_{c}\right)$ then is equal to

$$
\widehat{U}^{3 g}\left(\theta ; \gamma_{c}\right)=I^{2 g}-\left(\widehat{M}_{2 h}^{2 g}\left(2 \theta_{\beta}\right)\right)^{\gamma_{c}} .
$$

The spectral radius of the error transformation operator gives a prediction of the asymptotic rate of convergence of the multigrid method. This asymptotic convergence is expressed in terms of the asymptotic convergence factor per cycle, which is equal to

$$
\mu=\sup _{\theta \in \Theta_{3 g} \backslash \Psi_{3 g}} \rho\left(\widehat{M}^{3 g}(\theta)\right),
$$

with $\rho$ is the spectral radius. A requirement for convergence of the multigrid algorithm is that the spectral radius satisfies the condition $\mu<1$. By minimizing the spectral radius of the three-level multigrid error transformation operator (9), we obtain optimized multigrid algorithms. 


\section{Optimizing multigrid for space-time DG discretizations}

The theory of the previous sections holds for general linear discretizations and smoothing operators, but in this paper we are specifically interested in designing optimized multigrid methods for higher order accurate space-time DG discretizations. For the optimization, we will consider the $2 \mathrm{D}$ advection-diffusion equation as model problem

$$
\partial_{t} u+\mathbf{a} \cdot \nabla u-\nabla \cdot(\overline{\bar{A}} \nabla u)=0, \quad x \in \Omega \subset \mathbb{R}^{2}, t \in \mathbb{R}^{+},
$$

where we assume that the advection velocity $\mathbf{a} \in \mathbb{R}^{2}$ and diffusion matrix $\overline{\bar{A}} \in\left(\mathbb{R}^{+}\right)^{2}$ are constant, with $\overline{\bar{A}}_{11}=v_{x}, \overline{\bar{A}}_{22}=v_{y}$ and $\overline{\bar{A}}_{12}=\overline{\bar{A}}_{21}=0$. We do not discuss the details of the space-time DG discretization for the advection-diffusion equation, but refer to [3,5] for more details. In the multigrid optimization we consider a uniform space-time mesh with elements $\Delta t \times \Delta x \times \Delta y$ and periodic boundary conditions. The discretization depends on the following dimensionless numbers:

$$
C F L=\frac{a \Delta t}{h}, \quad R e_{x}=\frac{a(\Delta x)^{2}}{v_{x} h}, \quad R e_{y}=\frac{a(\Delta y)^{2}}{v_{y} h}, \quad A R=\frac{\Delta y}{\Delta x},
$$

in which $h=\Delta x \sqrt{1+A R^{2}}$ and $a=\sqrt{a_{x}^{2}+a_{y}^{2}}$. Furthermore, we introduce the flow angle $\gamma^{\text {flow }}$ with respect to the $x$-axis so that $a_{x}=\cos \left(\gamma^{\text {flow }}\right) a$ and $a_{y}=\sin \left(\gamma^{\text {flow }}\right) a$.

\subsection{Pseudo-time integration and Runge-Kutta methods}

The system of algebraic equations resulting from the space-time DG discretization of the $2 \mathrm{D}$ advection-diffusion equation can be represented as

$$
\mathscr{L}\left(\hat{u}^{n} ; \hat{u}^{n-1}\right)=0,
$$

with $\hat{u}^{n}$ the expansion coefficients of a polynomial approximation of $u$ and $n$ refers to the time index. To solve the system of coupled equations for the expansion coefficients $\hat{u}^{n}$ in (12), a pseudo time derivative is added to the system [7]:

$$
\Delta x \Delta y \frac{\partial \hat{u}^{*}}{\partial \tau}=-\frac{1}{\Delta t} \mathscr{L}\left(\hat{u}^{*} ; \hat{u}^{n-1}\right),
$$

which is integrated to steady-state in pseudo-time. At steady state, $\hat{u}^{n}=\hat{u}^{*}$. For the pseudo-time integration we introduce the dimensionless number $\lambda=\Delta \tau / \Delta t$ and use the pseudo-time $C F L$ number, defined as $C F L^{\tau}=\lambda C F L$. To solve (13) we consider $N$-stage Runge-Kutta methods. For notational purposes, we set $\mathscr{L}\left(\hat{V}^{*} ; u^{n-1}\right)=$ $\mathscr{L}\left(\hat{V}^{*}\right)$. Initialize $\hat{V}^{0}=\hat{u}^{n-1}$. Then, an $N$-stage Runge-Kutta scheme is given by: 


$$
\left(1+\beta_{j} \lambda I\right) \hat{V}^{j}=\hat{V}^{0}-\lambda\left(\sum_{l=1}^{j} \alpha_{j+1, l} \mathscr{L}\left(\hat{V}^{l-1}\right) /(\Delta x \Delta y)\right)+\lambda \beta_{j} \hat{V}^{j-1}, \quad j=1, \ldots, N,
$$

with $\hat{u}^{*}=\hat{V}^{N}$. We see that there are a number of free parameters in the RungeKutta smoother. The smoother is therefore a good candidate for optimization. We will minimize the spectral radius (10) by optimizing the parameters $\alpha$ and $\beta$. In this paper only 5-stage Runge-Kutta schemes are considered for which we require that they are second order accurate in pseudo-time. This requirement gives constraints on the $\alpha$ coefficients. The $\beta$ coefficients serve as the Melson correction to improve stability for small values of $\lambda \cong 1$, see Melson et al. [4].

\subsection{Optimization results}

We now provide some examples of the optimization of the Runge-Kutta (RK) smoothers for multigrid. We distinguish between diagonal RK schemes (dRK5) and full RK schemes (fRK5) in which all coefficients $\alpha_{j+1, l}$, with $1 \leq l \leq j \leq N$, are nonzero. We present optimized RK coefficients for the second $(p=1)$ and third $(p=2)$ order accurate space-time DG discretizations of the $2 \mathrm{D}$ advection-diffusion equation. For this we use the optimization procedures fminsearch and fmincon, available in Matlab. As constraint in the fmincon procedure, we require that both the spectral radius of the smoother and the three-level multigrid error transformation operator are less than 1 . The optimization was performed for advection dominated steady flows in which we fix the Reynolds numbers $R e_{x}=R e_{y}=100$ and the $C F L$ number as $C F L=100$. We also set the flow angle $\gamma^{\text {flow }}=\pi / 4$, the aspect ratio $A R=1$ and the number of pre- and post-smoothing steps $v_{1}=v_{2}=1$. On the coarsest grid, we use four smoother steps instead of an exact inverse. Furthermore, $\gamma=1$. As initial guess in the optimization procedure, we use the EXI RK method [7] for the optimized dRK5 scheme. We then use the dRK5 scheme as initial guess to obtain the fRK5 scheme. The optimized coefficients and spectral radii of the smoother $\rho^{\mathscr{S}}$ and the 3-level multigrid operator $\rho^{M G}$ are given in Table 1. As a comparison, we also give the spectral radius of the 3-level multigrid operator with EXI-RK smoother, $\rho^{E X I-M G}$ when using the given parameters. We see that for these parameters the multigrid algorithm with the EXI smoother is very unstable, while good convergence can be achieved with our optimized schemes.

\section{Testing multigrid performance}

In this section we test the multigrid performance. We start in Section 5.1 by comparing the optimized $h$-multigrid algorithms of the previous sections to the original EXI-EXV $h$-multigrid method [3]. For this we consider the 2D advection-diffusion equation. In Section 5.2 we consider a more complex test case in which we solve 
Table 1 Optimized coefficients for the dRK5 and fRK5 smoothers for 3-level multigrid for steady flows.

\begin{tabular}{|c|c|c|c|c|}
\hline & $\mathrm{dRK} 5 p=1$ & fRK5 $p=1$ & dRK5 $p=2$ & fRK5 $p=2$ \\
\hline$\alpha_{21}$ & 0.05768995298 & 0.0578331573 & 0.04865009589 & 0.04877436325 \\
\hline$\alpha_{31}$ & - & -0.0002051554736 & - & -0.0002188348438 \\
\hline$\alpha_{32}$ & 0.1405960888 & 0.1403808301 & 0.130316854 & 0.1300906122 \\
\hline$\alpha_{41}$ & - & 0.0003953470071 & - & $2.608884832 \mathrm{e}-05$ \\
\hline$\alpha_{42}$ & - & -0.001195029164 & - & $2.444376496 \mathrm{e}-05$ \\
\hline$\alpha_{43}$ & 0.267958213 & 0.2681810517 & 0.2729621396 & 0.2734805705 \\
\hline$\alpha_{51}$ & - & 0.0001441249202 & - & -0.001250385487 \\
\hline$\alpha_{52}$ & - & -0.0002608610327 & - & -0.0007838720635 \\
\hline$\alpha_{53}$ & - & -0.0003368070181 & - & -0.0004890887712 \\
\hline$\alpha_{54}$ & 0.5 & 0.8473374098 & 0.5 & 4.412139367 \\
\hline$\alpha_{61}$ & - & 0.4115573097 & - & 0.8097217358 \\
\hline$\alpha_{62}$ & - & -0.003144851878 & - & 0.08435089009 \\
\hline$\alpha_{63}$ & - & -0.0001096455683 & - & -0.01986799007 \\
\hline$\alpha_{64}$ & - & 0.001555741114 & - & 0.01359815476 \\
\hline$\alpha_{65}$ & 1.0 & 0.5901414466 & 1.0 & 0.1121972094 \\
\hline$\beta_{1}$ & 0.05768995298 & 0.04887040625 & 0.04865009589 & 0.5551936269 \\
\hline$\beta_{2}$ & 0.1405960888 & 0.1274785795 & 0.130316854 & 0.1333199239 \\
\hline$\beta_{3}$ & 0.267958213 & 0.2287556298 & 0.2729621396 & -1.332263675 \\
\hline$\beta_{4}$ & 0.5 & 0.9547064029 & 0.5 & -3.649588578 \\
\hline$\beta_{5}$ & 1.0 & 2.52621971 & 1.0 & 0.46771792 \\
\hline$C F L^{\tau}$ & 0.8 & 0.8 & 0.4 & 0.4 \\
\hline$\rho^{\mathscr{S}}$ & 0.98812 & 0.98914 & 0.98974 & 0.9896 \\
\hline$\rho^{M G}$ & 0.89151 & 0.81762 & 0.90049 & 0.89903 \\
\hline$\rho^{E X I-M G}$ & 167.06 & - & 124.02 & - \\
\hline
\end{tabular}

the Euler equations for inviscid flow over an NACA0012 airfoil. We will compare the performance of $h$-multigrid with $p$ - and $h p$-multigrid.

\subsection{The 2D advection-diffusion equation}

In order to demonstrate the performance of the optimized algorithms we consider (11) on $\Omega=(0,1)^{2}$ with initial condition $u(x, y, 0)=1-\frac{1}{2}(x+y)$ and boundary condition $u(x, y, t)=g(x, y)$. Here $g(x, y)$ equals at the domain boundary the exact steady state solution of (11) given by:

$$
u(x, y)=\frac{1}{2}\left(\frac{\exp \left(a_{1} / v_{x}\right)-\exp \left(a_{1} x / v_{x}\right)}{\exp \left(a_{1} / v_{x}\right)-1}+\frac{\exp \left(a_{2} / v_{y}\right)-\exp \left(a_{2} y / v_{y}\right)}{\exp \left(a_{2} / v_{y}\right)-1}\right) .
$$

In the discretization we use a Shishkin mesh [3] which is suitable for dealing with boundary layers. The parameters in the test cases are the following: we consider a mesh with $32 \times 32$ elements, one physical time step, with $\Delta t=100, a=\sqrt{2}$, $v_{x}=v_{y}=0.01$ and a flow angle $\gamma^{\text {flow }}=\pi / 4$. For the optimized RK schemes, we 


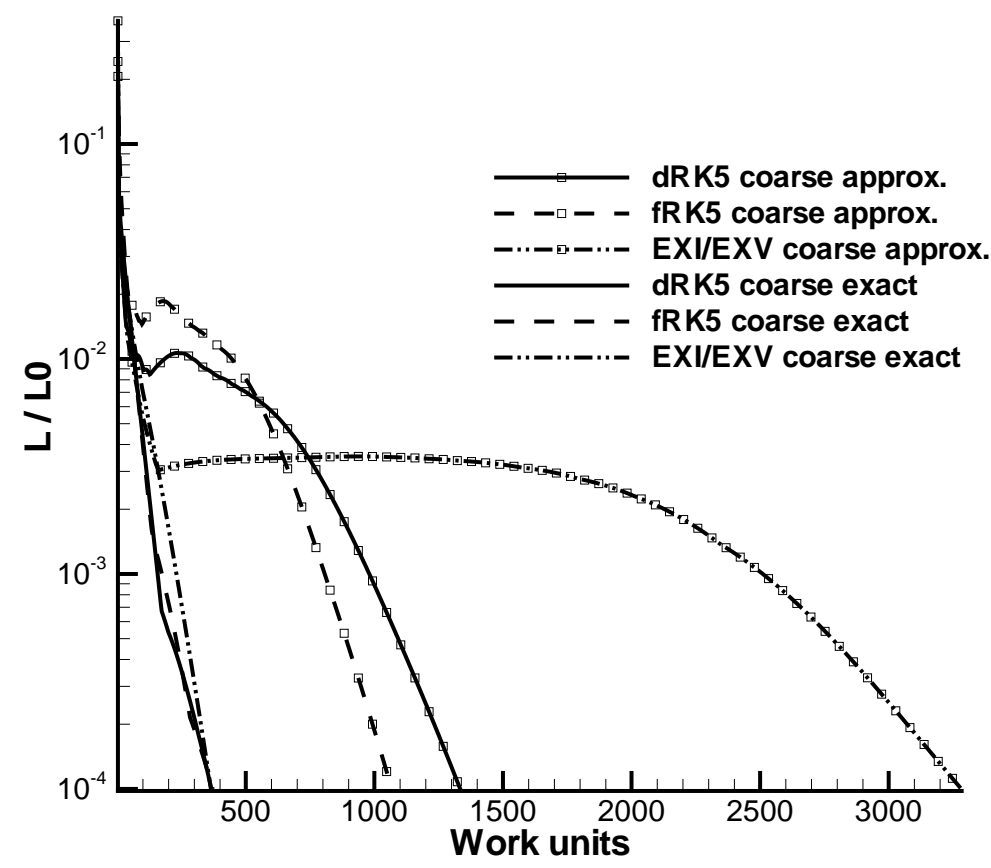

Fig. 1 Convergence results of second order space-time DG for three level multigrid algorithms with different Runge-Kutta smoothers. (dRK5, fRK5 and the EXI-EXV scheme [2], exact and approximate solution of equations on coarsest mesh).

used a local pseudo-time scaling to deal with viscous flows [9]. For the multigrid computations we use $v_{i}=1, i=1,2,3,4$ and $\gamma=1$. On the coarsest mesh we investigate the effect of using $v_{C}=4$ smoother iterations or solving the discrete system exactly.

In Figures 1 and 2, we show the convergence results of the different smoothers for 3-level multigrid. We see that in all cases a big improvement is obtained with the optimized Runge-Kutta smoothers over the original EXI-EXV smoother. For a second order accurate space-time DG discretization the number of multigrid cycles to obtain 4 orders of reduction in the residual is reduced from 3283 to 371. For the third order accurate DG discretization the number of multigrid cycles reduces from 21254 to 184. Furthermore, comparing dRK5 with fRK5, we see that the differences for a second order accurate space-time DG discretization is negligible. For a third order accurate space-time DG discretization this difference is, however, significant. Using more Runge-Kutta coefficients enlarges the possibilities to optimize the smoother. 


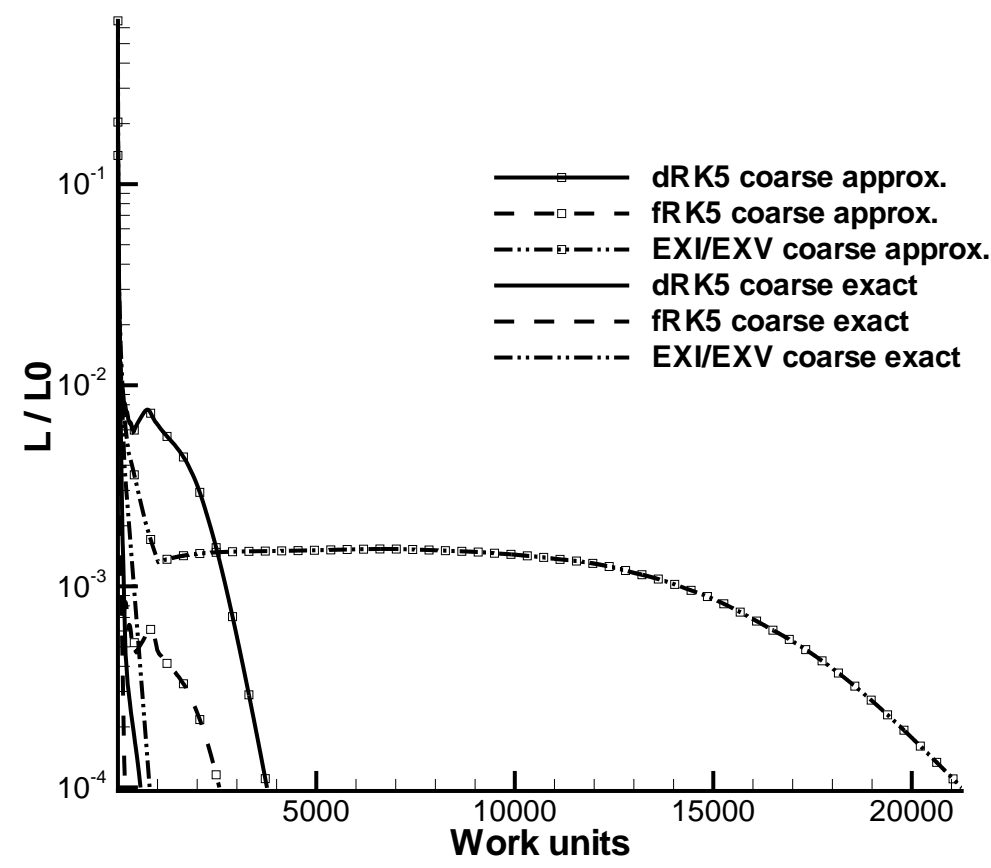

Fig. 2 Convergence results of third order space-time DG for three level multigrid algorithms with different Runge-Kutta smoothers. (dRK5, fRK5 and the EXI-EXV scheme [2], exact and approximate solution of equations on coarsest mesh).

The effect of solving the equations on the coarsest mesh with high accuracy is very large. Without this the multigrid convergence significantly slows down after a rapid initial decrease of the residual. In particular, for nonlinear problems it is tempting to solve the algebraic system on the coarsest mesh only approximately, because otherwise a global Newton solver is required. The effect of accurately solving the algebraic equations for the linear advection-diffusion equation on the coarsest mesh is, however, non-negligible.

\subsection{The Euler equations}

We now compare the performance of an $h$-multigrid method with $p$ - and $h p$ multigrid. Since the difference between EXI and the optimized RK smoothers for the Euler equations is small we will only show the EXI results. As test case we consider 2D steady subsonic flow around a NACA0012 airfoil with an angle of attack of 
$\alpha=2^{\circ}$ and far-field Mach number $M a=0.5$ (MTC1 test case). Since this test case is a steady-state flow problem, we consider a space-time DG discretization which is only first-order accurate in time but third-order accurate in space. The grid around the airfoil has $448 \times 64$ elements.

For single-grid, $p$ - and $h p$-multigrid computations we used a pseudo-time CFL number of $C F L^{\tau}=1.6$, while for $h$-multigrid $C F L^{\tau}=0.8$. Larger pseudo-time CFL numbers for $h$-multigrid resulted in unstable calculations. For the $p$-multigrid method we solve the lowest order problem approximately taking $v_{C}=20$. For the $h$ and $h p$-multigrid methods we solve the coarsest grid problem approximately, also taking $v_{C}=20$. Furthermore, for the $h$-multigrid method, we also solve the coarse grid problem exactly using a matrix-free Newton method. In all cases, 5 pre- and post-smoothing steps were taken on each multigrid level. The Mach contours are given in Figure 3 while the convergence history plot is given in Figure 4.

We see that $h$-multigrid performs the worst while $p$ - and $h p$-multigrid converge six orders in approximately the same amount of work units. We, however, had to take a twice as small $C F L^{\tau}$ number in the $h$-multigrid calculation compared to the other calculations. Furthermore, we see that after the high-frequency error modes have been smoothed, $h$-multigrid efficiency quickly deteriorates. A possible reason for this could be that the coarse-grid problem of the $h$-multigrid algorithm is not solved well with respect to the characteristic components, see [12]. We also see that there is hardly any difference in solving the coarse grid equations exactly with the Newton method or approximately by performing $v_{C}$ smoothing steps. This in contrary to the results obtained in Section 5.1, where we saw a large improvement when the coarse grid problem was solved exactly.

Regarding the $h p$-multigrid, where we first start with $p$-multigrid and continue at the lowest polynomial order with $h$-multigrid, we see that initially there is a significant improvement in reduction of the residual compared to the single-grid computation, but in the asymptotic regime single-grid and $h p$-multigrid have approximately the same residual reduction per work unit. The reason for this behavior is unclear yet. For the $p$-multigrid method, initial convergence is significantly faster than for the single-grid computations, but in the asymptotic regime also a comparable convergence history is obtained.

\section{Conclusions}

Using discrete Fourier analysis, we have analyzed two- and three-level multigrid algorithms for the solution of linear algebraic systems originating from higher order accurate space-time DG discretizations. For the 2D advection-diffusion equation we have shown that by minimizing the spectral radius of the multigrid error transformation operator, a significant improvement in the multigrid performance can be achieved. The algorithms have been tested on a $2 \mathrm{D}$ problem containing boundary layers, where the optimized Runge-Kutta smoothers show a significant improvement compared to the original EXI-EXV Runge-Kutta smoother discussed in [2,3]. 


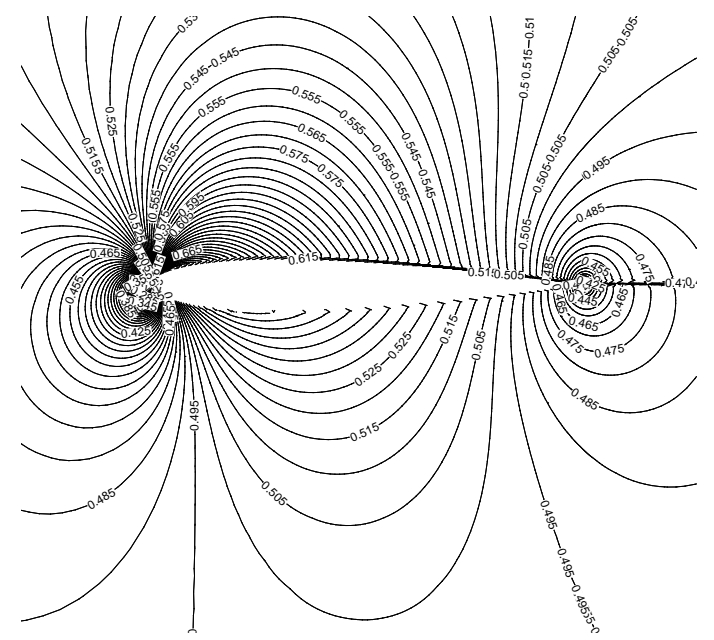

Fig. 3 Mach contours of inviscid flow around an NACA0012 airfoil $\left(\alpha=2^{\circ}, M a=0.5\right)$.

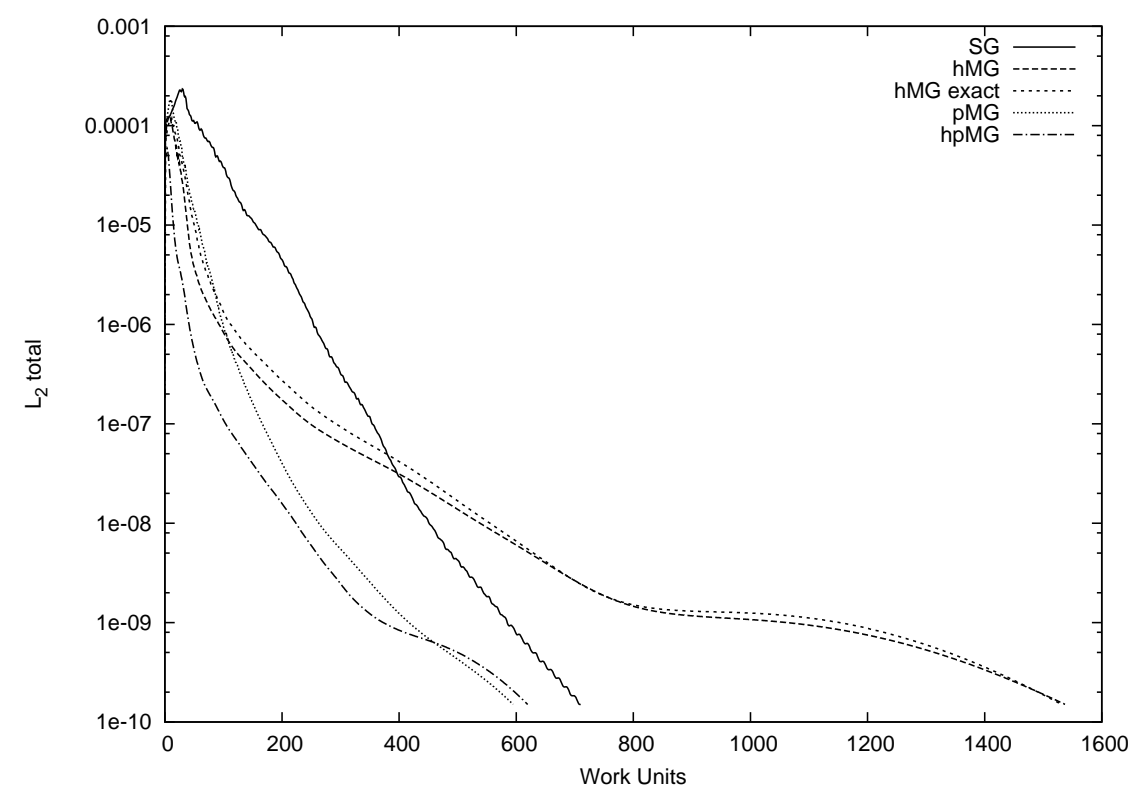

Fig. 4 Convergence history of single-grid, $h$-, $p$ - and $h p$-multigrid techniques for the solution of inviscid flow around an NACA0012 airfoil $\left(\alpha=2^{\circ}, M a=0.5\right)$. 
Apart from optimizing the multigrid smoother, also the solution of the algebraic system on the coarsest mesh has a big impact on the multigrid performance.

We also compared the performance of $h$-multigrid with $p$ - and $h p$-multigrid for solving the Euler equations. We considered subsonic inviscid flow around a NACA0012 airfoil. No significant difference was observed between the EXI scheme and the optimized Runge-Kutta smoothers. The main problem is the deterioration of the convergence rate after the high frequency error modes are smoothed, in particular for $h$-multigrid. Also, the effect of solving the equations on the coarsest mesh exactly or approximately is small. This in contrast with the $2 \mathrm{D}$ advection-diffusion case. Furthermore, we saw that the $p$ - and $h p$-multigrid methods show a better convergence rate than the $h$-multigrid method.

Acknowledgements This research was partly funded by the ADIGMA project which was executed in the 6th Research Framework Work Programme of the European Union within the Thematic Programme Aeronautics and Space.

\section{References}

1. Brandt, A.: Rigorous quantitative analysis of multigrid, I: Constant coefficients two-level cycle with $L_{2}$-norm. SIAM J. Numer. Anal. 31, 1695-1730 (1994).

2. Klaij, C.M., van der Vegt, J.J.W., van der Ven, H.: Pseudo-time stepping methods for spacetime discontinuous Galerkin discretizations of the compressible Navier-Stokes equations. J. Comput. Phys. 219, 622-643 (2006).

3. Klaij, C.M., van Raalte, M.H., van der Ven, H., van der Vegt, J.J.W.: $h$-Multigrid for spacetime discontinuous Galerkin discretizations of the compressible Navier-Stokes equations. J. Comput. Phys. 227, 1024-1045 (2007).

4. Melson, N.D., Sanetrik, M.D., Atkins, H.L.: Time-accurate Navier-Stokes calculations with multigrid acceleration. in Proc. 6th Copper Mountain Conference on multigrid methods, 423437, (NASA Langley Research Center 1993)

5. Sudirham, J.J., van der Vegt, J.J.W., van Damme, R.M.J.: Space-time discontinuous Galerkin method for advection-diffusion problems on time-dependent domains. Appl. Numer. Math. 56, 1491-1518 (2006).

6. Trottenberg, U., Oosterlee, C.W., Schüller, A.: Multigrid. Academic Press, London (2001)

7. van der Vegt, J.J.W., van der Ven, H.: Space-Time Discontinuous Galerkin Finite Element Method with Dynamic Grid Motion for Inviscid Compressible Flows I. General Formulation. J. Comput. Phys. 182, 546-585 (2002).

8. van der Vegt, J.J.W., Rhebergen, S.: Multigrid optimization using discrete Fourier analysis. Von Karman Institute Lecture Notes (2009)

9. van der Vegt, J.J.W., Rhebergen, S.: Multigrid optimization for higher order accurate spacetime discontinuous Galerkin discretizations, in preparation

10. Wesseling, P.: An introduction to multigrid methods. Wiley, Chicester (1991).

11. Wienands, R., Joppich, W.: Practical Fourier analysis for multigrid methods. Chapman \& Hall/CRC (2005).

12. Yavneh, I.: Coarse-grid correction for nonelliptic and singular perturbation problems. SIAM J. Sci. Comput. 19, 1682-1699 (1998). 